\title{
Research on Test System for Disease Aided Diagnosis
}

\author{
Wei CHEN" ${ }^{a}$, Zhangxiong WEN, Lin CHEN and Fengwen SUN \\ School of Electrical Engineering and Intelligentization, Dongguan University of Technology, 523808 Dongguan, Guangdong Province, \\ P.R.China
}

\begin{abstract}
A real-time multi parameter acquisition system is designed in this paper. Sensors collect physiological parameter data. The parameters are transmitted to the main processor module via a wireless sensor network. The main processor processes the data. The results reflect the current physiological condition of the monitored person.If the physiological condition is abnormal, alarm measures will be initiated to inform the monitor. Wavelet analysis method is used to denoise data. The wavelet transform and least squares support vector machines are used to predict the information, and the model is corrected by the statistical results of the measured data. Experiments show that the modified method is effective.
\end{abstract}

\section{Introduction}

People pay more and more attention to the condition of health in daily life. Many people are in a state of subhealth now. For some elderly people, physical functioning and limb flexibility are falling. Real time monitoring is needed to prevent common geriatric diseases, such as stroke, asthma, coronary heart disease and some other chronic diseases.

Health management originally originated in the United States, Canada and so on. These western countries carried out the public disease prevention service firstly. People with different health conditions have different health needs. Health management performs different tests, analyses and assessments for different people. Health management provides professional health advice and services to the population and proposes appropriate health management plans. Health management can coordinate individual and social actions, and then carry out systematic management according to various risk factors. At present, the object of health management is limited, in addition to high-income groups in China. Most of the residents have no sense of health management, especially the young workers who work hard in the city. So the public awareness is still very low[1].

The occurrence and development of diseases, especially chronic diseases, can be interfered. From the point of view of health management, everyone experiences the process from health to disease. Generally it is from the health state, to a low risk state, and then to a high risk state, and then to the onset of disease, and finally is the clinical state. The course of chronic disease can be very long. It usually takes a few years or more years, or even decades. But one thing in common is that they are preventable and controllable. Chronic diseases change in a longer process and are not easily detected. However, the risk factors of disease can be found through the examination and analysis of health management system. People can carry out targeted prevention before the disease formation.

Health management can prevent and control the occurrence and development of disease. It can also reduce the cost of medical treatment and improve the quality of life. Health management helps people carry out health management education, improve people's health management consciousness and level, and understand the related threat health factors in life. We can continue to improve our health through intervention, testing, evaluation, and information collection.

The two important links in health management are the collection and detection of health information. Health management can only be carried out effectively according to the adjustment of the data obtained from the collection and testing of health information

In this paper, a portable device is designed to monitor the physiological parameters of human body in real time. These parameters include pulse rate, oxygen, body temperature, and so on. ZigBee technology is used to realize data communication in the network. The system is based on the self formed wireless sensor network. Sensors are worn on the observer. The terminal node collects the physiological parameters of the monitored person. Parameters are transmitted to the coordinator in real time. The coordinator sends data to the master processor via the interface. The system facilitates realtime perception of physical parameters of the body.

The premise of finding a disease is to obtain accurate physiological information. In physiological signal analysis, the useful signals are usually low frequency signals or some relatively stationary signals, while the noise signals usually show high frequency

a Corresponding author: grace_chen@163.com 
signals. In this paper, the method of removing noise from physiological signals is studied, and the noise and interference signals contained in physiological parameters are effectively suppressed by using wavelet transform.

The physiological parameters of the monitoring object are analyzed, and the hidden slow changes are found. It can predict the disease of the monitoring object and notify observers in time to prevent it. In this paper, comprehensive prediction method is adopted to realize the analysis of multiple physiological parameters of human body. Statistical method is used to analyze the actual data, which is used to correct the prediction results so as to improve the prediction accuracy.

\section{System Overall Design}

Physiological parameter acquisition is realized by sensor module. The collected data is transmitted to the Zigbee terminal node. The terminal node transmits the coordinator to the coordinator after the network is completed, and realizes the wireless transmission of data[2]. After receiving the physiological parameters sent by the Zigbee terminal node, the Zigbee coordinator sends it to the upper computer for data processing. The structure of system is shown in Figure 1.

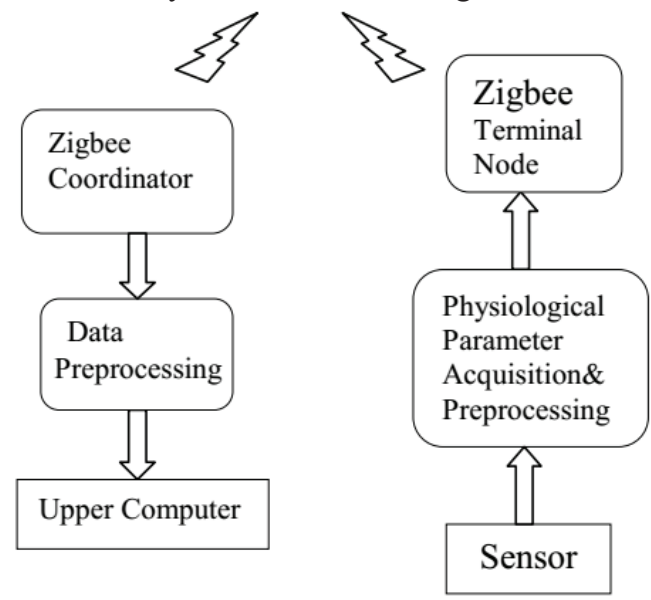

Figure 1. System Overall Structure.

The architecture of the upper computer software consists of three parts: the acquisition end, the display interface and the storage end.

The data acquisition process at the acquisition end is: data reception, identity authentication, data acquisition and data transmission.

Some of the details of the acquisition process are as follows:

(1) The acquisition end of physiological parameters uses C\# to develop multi thread program, and the acquisition end asynchronously receives data through the serial port. If the data is read by the serial port, the data is parsed. When the string conforms to the agreed string format, asynchronous calls are made to the parsed data. The data will be saved to the database, and the line graph can be automatically drawn from the display interface of the main interface.

(2) When the physiological parameter data is collected, the the acquisition end of user creates the
thread.Functions are constructed in the singleton mode to complete the new and start of the thread.

(3) The opening and closing of the serial port is realized by the way of asynchronous delegate, and the time interval of the acquisition can be chosen as one of "05 seconds", "30 seconds", "1 minutes" and "10 minutes".

(4) When the physiological parameters are imported into the acquisition system in the form of Excel, the Excel is opened by COM, and the data can be reached along the COM port. After the data is collected, the data line graph is redrawn.

The display interface is not only the operation interface of the whole software, but also the broken line display of physiological parameters. There is an operation selection on the display interface. To make the interface look more friendly, the left side of the main form interface is designed to display graphical small interface, the right side of the design is the operation of other users. When the user selects different physiological parameters, the left side of the small interface changes line graph. In the small interface, three physiological parameters are as follows: oxygen unit is $\%$, body temperature unit is degrees Celsius and pulse rate units are units / min..

Some of the details of the display interface are designed as follows:

(1) In the small parameter display interface, the $X$ axis is the number of measurements, and the $\mathrm{Y}$ axis is the measured data. When the new data is collected, move the old data forward a bit by using an asynchronous delegate call firstly, and then filled with new data to the bottom, then redraw the data line chart based on the current data. When drawing, the logical coordinate points are converted to the drawing coordinate point. The Y axis of the drawing coordinate point is from top to bottom, and the $\mathrm{X}$ axis is the offset to the left or to the right.

(2) The user operation is divided into software settings, view $\log$ entries, import data, export data and four physiological parameters selection, the software settings items and view log entries are used as actions to jump to the interface of another form

(3) A form interface is not enough to complete all the functions, so you need to create another two forms. In the new form interface, there are serial selection, serial access and closing items, baud rate entries, database selection, collection interval selection, and another new form has log information .

(4) The export and import of data are implemented using the COM call.

(5) The physiological parameter selection uses the Radio Button control. The conversion of physiological parameters is achieved through the Click event of the control. After the Radio Button is selected, the data line graph is redrawn immediately.

(6) The software settings item and the log entries also use the Button (button) control's Click event, which automatically jumps out of the form interface and log interface.

(7) In the form interface, the opening and closing of serial ports are Click events using Button (button) control, and are implemented asynchronously. The serial selection, baud rate, and collection interval selections use the 
ComboBox (drop-down combo box) control. The database selection is used to select the database where the data is stored.

The storage end is divided into two parts, one is the data stored in the Access 2010 database[3]. The other part is data storage to the Excel table. The difference is that the data is stored automatically when it is stored in the database, and the Excel table is selected by the user himself[4].

\section{Denoising of Physiological Signals Based on Wavelet Analysis}

The traditional design of physiological signal denoising uses filter design, but the denoising accuracy is difficult to satisfy. In recent years, denoising and recognition algorithms are mainly wavelet transform, adaptive filter and other methods. The basic idea of these algorithms is converting the signal into the frequency domain, partial inhibition of noise signals, and ECG signal recognition in the main frequency range; or denoising and recognition by using mathematical morphology and combined with the characteristics of ECG signal. Although these algorithms can guarantee better denoising effect and higher recognition accuracy, the algorithm is complex and difficult to meet the requirement of real-time signal processing.

The research in this paper is aimed at the actual needs of the project. This paper chooses wavelet analysis as the basic algorithm, because wavelet analysis has the characteristics of multi-resolution. Multi-resolution timefrequency domain analysis can focus on any signal details, and wavelet analysis has been successfully applied in many fields. This system adopts wavelet lifting, because it is a fast wavelet transform, which can greatly reduce the amount of computation; the weighted threshold shrinkage method is improved in this system, because it improves the denoising effect; the system uses intermediate denoising results combined with difference method. Denoising and recognition algorithm are combined to reduce computation complexity. The algorithm has the advantages of less computation and simple structure, so it is easy to implement by FPGA, and can meet the requirements of real-time processing of monitoring equipment.

\section{Research on Monitoring Information Prediction}

Prediction uses time as independent variable, uses monitoring data as dependent variable, and establishes a functional relation between monitoring information and time. The prediction uses the monitoring data at each moment, and uses regression model to predict the future physiological parameters.

Due to the shortage of existing monitoring information forecasting methods, this paper combines wavelet decomposition and least squares support vector machines to study prediction techniques to improve accuracy. Wavelet analysis has the characteristic of multi-resolution.

Support vector machines (SVM) have the advantages of small sample, nonlinear, high dimension, avoiding local minimum point and avoiding over learning phenomenon.Least squares support vector machine instead of inequality constraints with equality constraints. The algorithm can be transformed into linear equations, which choose less parameters than the standard support vector machine method, and no longer need to specify the precision of convergence criterion. The utility model has the advantages of simple operation, fast solving speed and high precision. The mathematical model is constructed by the combination of wavelet decomposition and least squares support vector regression. Then the model is used to predict the monitoring signal.

The original parameter sequence is decomposed into approximation signal and detail signal by wavelet decomposing. The forecasting model of approximation signal and detail signal can be establised. After getting the prediction value of the approximate signal and the detail signal, inverse wavelet transform is used on the prediction value of each part, and then the final prediction result is obtained.

The prediction process is broadly divided into the following steps: firstly, the monitoring data is normalized, which is to normalize the monitoring data before establish the prediction model, in order to reduce the computational complexity of numerical training process; secondly, wavelet decomposition is used to monitoring data normalized sequences in order to obtain the decomposed sequence of each layer ; the third is to construct training samples of each layer data after wavelet decomposition; the fourth is to calculate parameter of the model; parameters of training samples and the fourth step is then established with the third step training model; forecast model and forecast the trained data of each layer wavelet decomposition the inverse wavelet transform; the prediction results and data layer; anti normalization is to forecast model output data back Normalization operations are used to obtain the final prediction results.

then the trained prediction model is used to predict the data of each layer after wavelet decomposition; inverse wavelet transform is used to prediction results of each layer ; fnally, data inverse normalization is used, which is inverse normalization operation of the output data from the prediction model, and the final prediction results are obtained.

\section{Using Statistical Methods for Correction}

For the designed system, we use the statistical results of the measured data to correct the model.

Take the temperature test as an example to illustrate the effectiveness of the method. The system is tested with different temperatures. Test results are statistically calculated in the table, the difference between the test value and the standard value, and the relative law is analyzed. 
After more than 100 measurements and statistics, some results are shown in Figure 2, Figure 3, Figure 4.

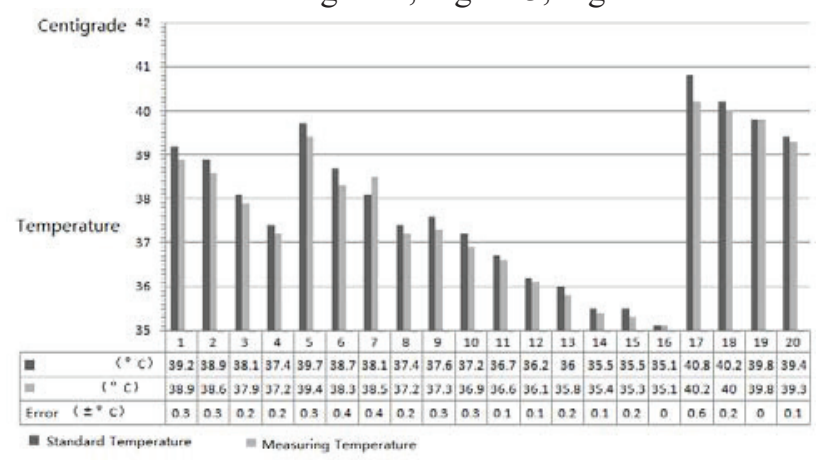

Figure 2. Comparison of Temperature.

From the 20 samples measured in Figure 2, it can be seen that the difference between the measured value and the standard temperature is maximum at $0.6{ }^{\circ} \mathrm{C}$ when in a high temperature state. The remaining temperature difference mainly falls at $0.1^{\circ} \mathrm{C}-0.3{ }^{\circ} \mathrm{C}$, and in a fever state, the temperature difference is larger.

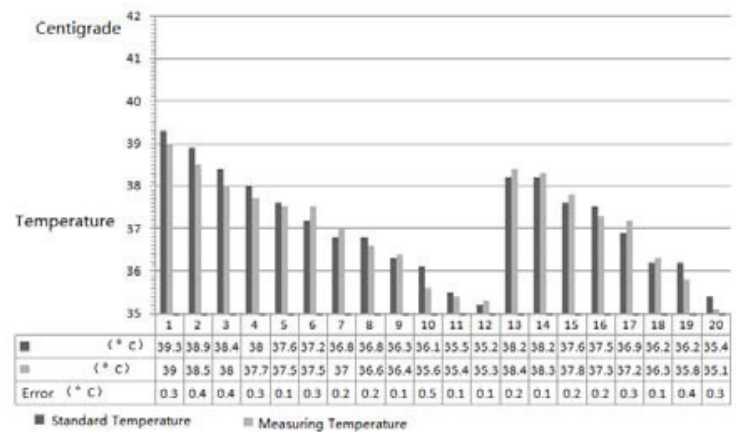

Figure3. Comparison of Temperature.

From the 20 samples measured in Figure 3, it can be seen that the difference between the measured value and the standard temperature is maximum at $0.5^{\circ} \mathrm{C}$ when in a lown temperature state. The remaining temperature difference mainly falls at $0.1^{\circ} \mathrm{C}-0.4^{\circ} \mathrm{C}$.

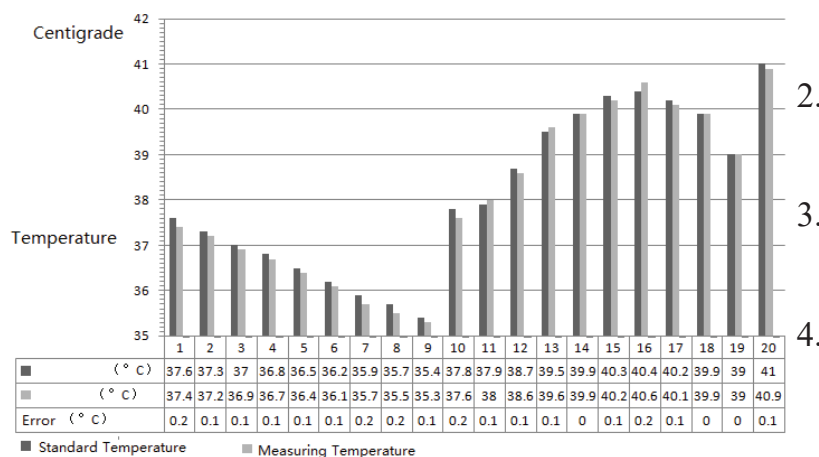

Figure4. Comparison of Temperature.

From the 20 samples measured in Figure 4, it can be seen that the difference between the measured value and the standard temperature is maximum at $0.4{ }^{\circ} \mathrm{C}$ when in a high temperature state. The remaining temperature difference mainly falls at $0.1^{\circ} \mathrm{C}-0.3{ }^{\circ} \mathrm{C}$. Compare Figure 4 and Figure 2. The difference between them is that Figure 4 is mainly in the state of rising temperature, and Figure 2 is mainly in the process of temperature drop.

By comparing the above values, the difference between the measured and the standard values can be obtained. In general, the data measured by the electronic probe is lower than the standard data. When the temperature is high, which is greater than $38^{\circ} \mathrm{C}$, the error of temperature probe is larger relatively.

Most of the errors are at 0.1 degrees $C, 0.2$ degrees $C$, 0.3 degrees $\mathrm{C}$, and 0.4 degrees $\mathrm{C}$. As the temperature decreases, the temperature error reduces slowly ${ }^{\circ} \mathrm{C}$. When the body temperature is lower than $38{ }^{\circ} \mathrm{C}$, the error is concentrated at $\pm 0.1{ }^{\circ} \mathrm{C}$ and $\pm 0.2{ }^{\circ} \mathrm{C}$.

The system adopts two times Fitting Correction System model. When the standard temperature is greater than or equal to $38^{\circ} \mathrm{C}$, the temperature error measured by the temperature probe is $\pm 0.2^{\circ} \mathrm{C}$. The error is $\pm 0.1{ }^{\circ} \mathrm{C}$ when the temperature is lower than $38{ }^{\circ} \mathrm{C}$. Therefore, after the model is corrected, the measurement accuracy is improved.

\section{Summary}

A real-time physiological parameter acquisition system is designed in this paper. The data is denoised by wavelet analysis, and wavelet decomposition and least squares support vector machines are used to predict the information. The model is corrected by statistical results, and the accuracy of the results is improved.

\section{Acknowledgment}

This research was financially supported as follows:

Guangdong science and technology project 2015A010103023;

Dongguan social science and technology development project 2013108101009.

\section{References}

1. Hong Jingchun, Community health education in the prevention and treatment of chronic diseases [J], Contemporary medicine, 12 :161-162 (2015)

2. Yang Xiuyu,Design of multi physiological parameter acquisition and management system [D],Beijing Jiaotong University( 2015)

3. Shi Xingjia, Wang Binghong, Access 2010 database application basic tutorial(Tsinghua University press, Beijing(2013)

4. Lei Lei, Wang Li,etc,Establishment of pain patient information management system using Access 2010 database $[\mathrm{J}]$, Chinese Journal of pain medicine, 22) :927-931(2016) 